\title{
Assessment the area of accretion curtains from fast aperiodic time variability of Intermediate Polars
}

\author{
Andrey Semena* \\ Space Research Institute RAS, Moscow, Russia \\ E-mail: san@iki.rssi.ru
}

Mikhail Revnivtsev

Space Research Institute

E-mail: revnivtsevaiki.rssi.ru

\section{David Buckley}

South African Astronomical Observatory

E-mail: dibnobesaao.ac.za

\begin{abstract}
Results of a study of the fast timing variability of the magnetic cataclysmic variable (mCV) EX Hya are presented. It was shown that one may expect the rapid flux variability of mCVs to be smeared out at timescales shorter than the cooling time of hot plasma in the post shock region of the accretion curtain near the WD surface. Estimates of the cooling time and the mass accretion rate, thus provide us with a tool to measure the density of the post-shock plasma and the crosssectional area of the accretion funnel at the WD surface. We have probed the high frequencies in the aperiodic noise of one of the brightest mCV EX Hya with the help of optical telescopes, namely SALT and the SAAO $1.9 \mathrm{~m}$ telescope. We place upper limits on the plasma cooling timescale $\tau<0.3 \mathrm{sec}$, on the fractional area of the accretion curtain footprint $f<1.6 \times 10^{-4}$, and a lower limit on the specific mass accretion rate $\dot{M} / A>3 \mathrm{~g} \mathrm{sec}^{-1} \mathrm{~cm}^{-2}$. We show that measurements of accretion column footprints via eclipse mapping highly overestimate their areas. From the measurements we estimate an upper limit to the penetration depth of the accretion disk plasma at the magnetosphere boundary $\delta r / r<10^{3}$
\end{abstract}

The Golden Age of Cataclysmic Variables and Related Objects - III, Golden2015

7-12 September 2015

Palermo, Italy

* Speaker.

${ }^{\dagger}$ A footnote may follow. 
Intermediate polars is compact binary systems with magnetic white dwarfs (WD) as an accretor. In IPs the accretion disk is partially destroyed by the magnetic field of the white dwarf in its vicinity. On the disk inner edge the matter is captured by the WD magnetic field and continue to fall onto its surface through the magnetically channeled flow, which usually referred as "accretion columns" (see, e.g. Ghosh \& Lamb, 1978). The geometry and size of the accretion column define the energy release processes of the falling matter. In turn the accretion column geometry, namely width of the accretion curtain, depends on the interaction process between the white dwarf magnetic field and the accretion disk matter. It follows that from the knowledge of the accretion column geometry we can obtain additional information about the magnetic properties of the accretion disk.

Processes of the energy release in Intermediate Polars and Polars are known - an accreted matter is decelerated and heated up in a stationary shock wave near the white dwarf surface. Heated plasma located between the white dwarf surface and the shock wave is cooling down due to an optically thin bremsstrahlung emission (see, e.g. Aizu, 1973). It was shown by Langer et al. (1981) that the shock wave position is unstable and should to oscillate. This, so called global thermal instability, leads to quasi periodical oscillations (QPOs) of the X-ray and optical fluxes in IPs and Polars. A characteristic time of that QPOs is defined by the matter density in the accretion column under the shock wave. Thus measurements of the QPOs frequencies is a convenient and easy way to study accreted matter properties near the WD surface. It should be noted, that such QPOs were found in several Polars, but not in any of known IPs.

An absence of the QPOs in IPs might be connected with the additional heating or cooling mechanisms, stabilizing the shock front position. Despite the absence of such QPOs in the observed light-curves of known IPs their luminosity variability still can be used to measure matter properties in the accretion curtain.

It is well known that the accretion flux near the white dwarf surface contains broad band variability spectrum Revnivtsev et al. (2010). We have shown qualitatively that the variability of the accretion flow should be smeared out on the time scale shorter than the plasma cooling time in the hot zone between the stationary shock wave and white dwarf surface. In order to check an expected variability suppression we performed 1D and 2D hydrodynamical simulations of the accretion processes in the accretion channel near the white dwarf surface. In these simulations the accretion flow with a predefined accretion rate $\dot{M}$ variability power spectrum fall down in the gravitation field on to the WD surface inside the channel with impenetrable walls. During the course of the simulation hot zone is formed, which produce freely escaping radiation via bremsstrahlung process, thus producing light-curve. The power spectrum of the obtained in the simulations light-curves corresponds to the power spectrum of the inflowing accretion rate $\dot{M}$ at the low frequencies and dumped at the high frequencies (see left panel of Fig. 1). Our simulations also show that the smearing of the variability take place exactly on the plasma cooling time scale, which is proportional to the specific accretion rate in the accretion column $\dot{M} / A$, where $A$ is accretion column footprint area and $\dot{M}$ is accretion rate on to the WD surface (Semena \& Revnivtsev, 2012). Thus the specific accretion rate for IPs can be measured by the analysis of a variability of their luminosity.

This approach was used to determine the specific accretion rate and characteristic size of the accretion channel for two well-known intermediate polars - EH Hya and LS Peg.

For EX Hya system, we analyzed power spectra of X-ray and optic lightcurves, which were obtained from the data of RXTE and XMM-Newton observatories in X-rays (Semena \& Revnivtsev, 
2014) and from data of 10-m and 1.9-m telescopes of the South African Astronomical Observatory (SAAO) in optics (Semena et al., 2014).

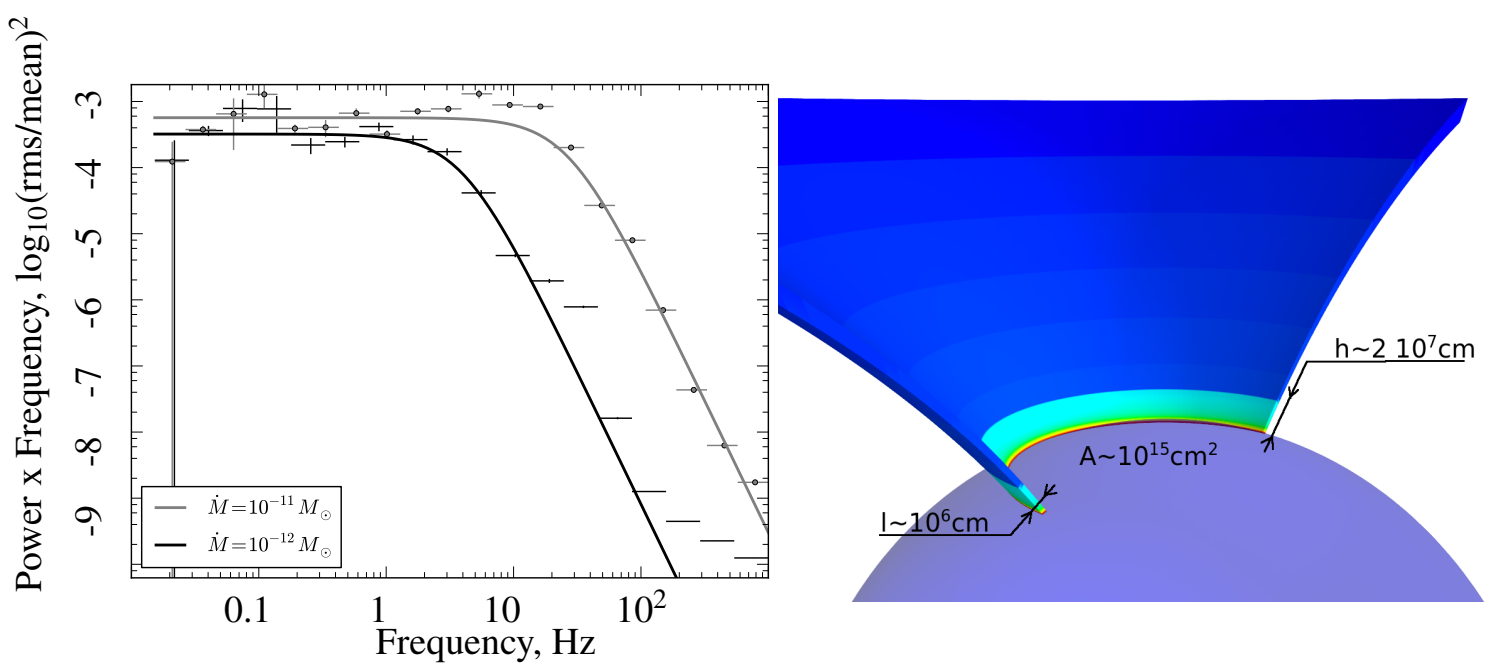

Figure 1: (left panel) Power spectra of the light curves produced in the hydrodynamic simulations with the different mean specific accretion rate. (right panel) Schematic view of the accretion flow near the white dwarf surface of EX Hya deduced from the analysis of its light curve variability

To obtain an intrinsic power spectrum of EX Hya we carefully took into account different background noise properties of observations carried out with different telescopes. We were able to extract the luminosity variability power spectrum of the EX Hya up to $5 \mathrm{~Hz}$ frequency with high signal to noise ratio. No prominent variability suppression was found in the received power spectrum.

From the obtained power spectrum we estimate a lower limit on the variability dumping frequency which in turn provides an upper limit on the specific accretion rate in the system $\dot{M} / A<$ $3 \mathrm{~g} / \mathrm{cm}^{2}$.

In a combination with the X-ray eclipse profiles (Mukai et al., 1998) obtained limit on the specific accretion rate allow to estimate the geometry of the accretion channel - e.g. determine its area $A \approx 10^{15} \mathrm{~cm}^{2}$, thickness $l \approx 10^{6} \mathrm{~cm}$, and height of the hot zone $h \approx 2 \times 10^{7} \mathrm{~cm}$.

\section{Conclusions}

We have shown that the accretion channel has a shape of a thin curtain with the footprint area $\approx 10^{15} \mathrm{~cm}^{2}$ and thickness only $\simeq 10^{6} \mathrm{~cm}$ (see right panel of Fig. 1 ).

This value of the accretion channel thickness is much smaller that the one usually used in the most of theoretical models. At the same time it is close to the estimates made in some theoretical works considered accretion disk interaction with the magnetic field (Lovelace et al., 1995; Campbell, 2010).

From the thickness of the accretion curtain we can estimate the thickness of the zone in the accretion disk where matter is captured by the WD magnetic field:

$$
\delta r=2 l\left(R_{\mathrm{in}} / R_{\mathrm{wd}}\right)^{3 / 2} \sqrt{1-R_{\mathrm{wd}} / R_{\text {in }}}
$$


where $\delta r$ is the thickness of the accretion curtain on the inner radius of accretion disk $R_{\mathrm{in}}, R_{\mathrm{wd}}$ is the white dwarf radius and 1 is accretion curtain thickness on the WD surface. Estimated thickness of the accretion curtain on the WD surface indicates that the zone in the accretion disk where the matter is captured by the magnetic field of the WD is also small $\delta r / r<10^{-3}$. Such thickness of this capturing zone indicates that the speed of the magnetic field propagation through the matter of the accretion disk is small and the dissipation of the magnetic field in the disk ineffective (see e.g. Campbell, 2010)

It is interesting to note that magnetically truncated disks in accreting binaries with neutron stars are similar to those in CVs. If we assume that the disk-magnetosphere coupling region at the boundary of the magnetosphere in neutron star binaries has similar thickness $\delta r / r<10^{-3}$ than we can estimate the fractional area of the neutron star accretion column: $A / 4 \pi r_{n s}^{2}<10^{5}$. Such a small fractional area of the accretion column should result in a very high density of the accreting matter near NS surface.

\section{References}

Aizu, K. 1973, Progress of Theoretical Physics, 49, 1184

Campbell, C.G. 2010, MNRAS, 403, 1339

Ghosh, P., \& Lamb, F. K. 1978, ApJ, 223, L83

Langer, S. H., Chanmugam, G., \& Shaviv, G. 1981, ApJ, 245, L23

Lovelace, R.V.E., Romanova, M.M., \& Bisnovatyi-Kogan, G. S. 1995, MNRAS, 275, 244

Mukai, K., Ishida, M., Osborne, J., Rosen, S., \& Stavroyiannopoulos, D. 1998, Wild Stars in the Old West, 137, 554

Revnivtsev, M., Burenin, R., Bikmaev, I., et al. 2010, A\&A, 513, A63

Semena, A.N., \& Revnivtsev, M.G. 2012, Astronomy Letters, 38, 321

Semena, A. N., \& Revnivtsev, M. G. 2014, Astronomy Letters, 40, 475

Semena, A. N., Revnivtsev, M. G., Buckley, D. A. H., et al. 2014, MNRAS, 442, 1123

\section{DISCUSSION}

Marina Romanova: 3-D simulations show that the hot spot is inhomogeneous, where the higher energy expected in the inner parts of the spots. Is it possible that the size of the spots is larger at low frequencies.

Andrey Semena: The temperature of the matter under the shock wave should be the same for inner and outer parts of the flow (therefore both parts should have same continuum energy spectra). The break in the power spectrum is determined by the part of the flow, which give the most part of the 
system luminosity - most dense inner regions. That mean that accretion curtain could have larger area, but most part of the accretion flux goes through the small cross-section area.

Pavel Kaigorodov: Is the curtains optically thin or thick?

Andrey Semena: Accretion curtain is optically thin under the shock wave $(\tau<0.3$ for the largest path under the shock wave) and also it is optically thin above the shock wave. 